\title{
Implicit Equations Involving the $p$-Laplace Operator
}

\author{
Greta Marino® and Andrea Paratore
}

\begin{abstract}
In this work we study the existence of solutions $u \in W_{0}^{1, p}(\Omega)$ to the implicit elliptic problem $f\left(x, u, \nabla u, \Delta_{p} u\right)=0$ in $\Omega$, where $\Omega$ is a bounded domain in $\mathbb{R}^{N}, N \geq 2$, with smooth boundary $\partial \Omega, 1<p<\infty$, and $f: \Omega \times \mathbb{R} \times \mathbb{R}^{N} \times \mathbb{R} \rightarrow \mathbb{R}$. We choose the particular case when the function $f$ can be expressed in the form $f(x, z, w, y)=\varphi(x, z, w)-\psi(y)$, where the function $\psi$ depends only on the $p$-Laplacian $\Delta_{p} u$. We also present some applications of our results.
\end{abstract}

Mathematics Subject Classification. 35R70, 35J92.

Keywords. Implicit elliptic problems, differential inclusions, $p$-Laplacian.

\section{Introduction and Main Results}

Let $\Omega \subset \mathbb{R}^{N}, N \geq 2$, be a bounded domain with smooth boundary $\partial \Omega$, let $1<p<\infty$, let $Y \subseteq \mathbb{R}$ be a nonempty interval possibly coinciding with $\mathbb{R}$, and let $f: \Omega \times \mathbb{R} \times \mathbb{R}^{N} \times \mathbb{R} \rightarrow \mathbb{R}$. In this paper, we shall consider the following implicit elliptic problem

$$
u \in W_{0}^{1, p}(\Omega), \quad f\left(x, u, \nabla u, \Delta_{p} u\right)=0 \quad \text { in } \Omega,
$$

where $\Delta_{p}$ denotes the $p$-Laplace operator, namely

$$
\Delta_{p} u:=\operatorname{div}\left(|\nabla u|^{p-2} \nabla u\right) \quad \forall u \in W^{1, p}(\Omega) .
$$

We consider the special case $f(x, z, w, y)=\varphi(x, z, w)-\psi(y)$, with $\varphi: \Omega \times \mathbb{R} \times$ $\mathbb{R}^{N} \rightarrow \mathbb{R}$ and $\psi: Y \rightarrow \mathbb{R}$. We require that $\psi$ depends only on $\Delta_{p} u$. We further distinguish among the case when $\varphi$ is a Carathéodory function depending on $x, u$, and $\nabla u$, and the case when $\varphi$ is allowed to be highly discontinuous in each variable. In this last case, the dependence on the gradient is no more allowed.

In both situations we first reduce problem (1.1) to an elliptic differential inclusion, but methods used are different and depend on the regularity of the function $\varphi$ and on the structure of the problem. 
More precisely, in the first case we make use of a result in [19] to obtain the inclusion

$$
-\Delta_{p} u \in F(x, u, \nabla u),
$$

where $F$ is a lower semicontinuous selection of the multifunction

$$
(x, z, w) \mapsto\{y \in Y: \varphi(x, z, w)-\psi(y)=0\} .
$$

A function $u \in W_{0}^{1, p}(\Omega)$ is called a (weak) solution to (1.2) if there exists $v \in L^{p^{\prime}}(\Omega), p^{\prime}$ being the conjugate exponent of $p$, such that $v(x) \in$ $F(x, u(x), \nabla u(x))$ for almost every $x \in \Omega$ and

$$
\int_{\Omega}|\nabla u|^{p-2} \nabla u \cdot \nabla w \mathrm{~d} x=\int_{\Omega} v w \mathrm{~d} x \quad \forall w \in W_{0}^{1, p}(\Omega) .
$$

We start with the general case $Y=\mathbb{R}$ and then we deduce, as a byproduct, the existence result when $Y$ is a closed interval of $\mathbb{R}$.

Existence of solutions to (1.2) is obtained by means of the following result, which is based on a selection theorem for decomposable-valued multifunctions, see $[2,13]$.

Theorem 1.1 (Theorem 3.1 of [17]). Let $F: \Omega \times \mathbb{R} \times \mathbb{R}^{N} \rightarrow 2^{\mathbb{R}}$ be a closedvalued multifunction. Suppose that

(h1) $F$ is $\mathcal{L}(\Omega) \otimes \mathcal{B}\left(\mathbb{R} \times \mathbb{R}^{N}\right)$-measurable;

(h2) for almost every $x \in \Omega$, the multifunction $(z, w) \mapsto F(x, z, w)$ turns out to be lower semicontinuous;

(h3) there exist $a \in L^{p^{\prime}}\left(\Omega, \mathbb{R}_{0}^{+}\right), b, c \geq 0$, with $\frac{b}{\lambda_{1, p}}+\frac{c}{\lambda_{1, p}^{1 / p}}<1$, such that

$$
\inf _{y \in F(x, z, w)}|y|<a(x)+b|z|^{p-1}+c|w|^{p-1} \quad \text { in } \Omega \times \mathbb{R} \times \mathbb{R}^{N} .
$$

Then, (1.2) has a solution $u \in W_{0}^{1, p}(\Omega)$.

Here, $\lambda_{1, p}$ is the first eigenvalue of the $p$-Laplacian in the space $W_{0}^{1, p}(\Omega)$.

The following is our main result, which extends [13, Theorem 3.2] to the case $p \neq 2$.

Theorem 1.2. Let $\varphi: \Omega \times \mathbb{R} \times \mathbb{R}^{N} \rightarrow \mathbb{R}$ be a Carathéodory function and let $\psi: \mathbb{R} \rightarrow \mathbb{R}$ be continuous. Suppose that

(i) $\psi$ is non-constant on intervals;

(ii) for all $(x, z, w) \in \Omega \times \mathbb{R} \times \mathbb{R}^{N}$, the function $y \mapsto \varphi(x, z, w)-\psi(y)$ changes sign;

(iii) there exist $a \in L^{p^{\prime}}\left(\Omega, \mathbb{R}_{0}^{+}\right), b, c \geq 0$, with $\frac{b}{\lambda_{1, p}}+\frac{c}{\lambda_{1, p}^{1 / p}}<1$, such that

$$
\sup \left\{|y|: y \in \psi^{-1}(\varphi(x, z, w))\right\}<a(x)+b|z|^{p-1}+c|w|^{p-1},
$$

for all $(x, z, w) \in \Omega \times \mathbb{R} \times \mathbb{R}^{N}$.

Then, there exists $u \in W_{0}^{1, p}(\Omega)$ such that

$$
\psi\left(-\Delta_{p} u\right)=\varphi(x, u, \nabla u) \quad \text { in } \Omega .
$$


When $\varphi$ is discontinuous we essentially follow [16, Theorem 3.1] to construct an appropriate upper semicontinuous multifunction $F$ related with $\psi^{-1}$ and $\varphi$, and then we solve the elliptic differential inclusion $-\Delta_{p} u \in F(x, u)$ using the following

Theorem 1.3 (Theorem 2.2 of [14]). Let $U$ be a nonempty set, let $\Phi: U \rightarrow$ $W_{0}^{1, p}(\Omega)$ and $\Psi: U \rightarrow L^{p^{\prime}}(\Omega)$ be two operators, and let $F: \Omega \times \mathbb{R} \rightarrow 2^{\mathbb{R}}$ be a convex closed-valued multifunction. Suppose that

$\left(i_{1}\right) \Psi$ is bijective and $v_{h} \rightarrow v$ in $L^{p^{\prime}}(\Omega)$ implies, up to subsequences, $\Phi\left(\Psi^{-1}\right.$ $\left.\left(v_{h}\right)\right) \rightarrow \Phi\left(\Psi^{-1}(v)\right)$ a.e. in $\Omega$. Furthermore, a non-decreasing function $g: \mathbb{R}_{0}^{+} \rightarrow \mathbb{R}_{0}^{+} \cup\{+\infty\}$ can be defined in such a way that

$$
\|\Phi(u)\|_{\infty} \leq g\left(\|\Psi(u)\|_{p^{\prime}}\right) \quad \forall u \in U ;
$$

$\left(i_{2}\right) F(\cdot, z)$ is measurable for all $z \in \mathbb{R}$;

$\left(i_{3}\right) F(x, \cdot)$ has a closed graph for almost every $x \in \Omega$;

$\left(i_{4}\right)$ There exists $r>0$ such that the function

$$
\rho(x):=\sup _{|z| \leq g(r)} d(0, F(x, z)), \quad x \in \Omega,
$$

belongs to $L^{p^{\prime}}(\Omega)$ and $\|\rho\|_{p^{\prime}} \leq r$.

Then, the problem $\Psi(u) \in F(x, \Phi(u))$ has at least one solution $u \in U$ satisfying $|\Psi(u)(x)| \leq \rho(x)$ for almost every $x \in \Omega$.

Extending [16, Theorem 3.1] to the case $p \neq 2$, we obtain the following result. We denote by $\pi_{0}$ and $\pi_{1}$ the projections of $\Omega \times \mathbb{R}$ on $\Omega$ and $\mathbb{R}$, respectively.

Theorem 1.4. Let $\mathcal{F}=\{A \subset \Omega \times \mathbb{R}: A$ is measurable and there exists $i \in$ $\{0,1\}$ such that $\left.m\left(\pi_{i}(A)\right)=0\right\}$, let $(\alpha, \beta) \subset \mathbb{R}$ be an interval which does not contain 0 , let $\psi:(\alpha, \beta) \rightarrow \mathbb{R}$ be continuous, let $\varphi: \Omega \times \mathbb{R} \rightarrow \mathbb{R}$, and let $p>N$. Suppose that

(i) $\varphi$ is $\mathcal{L}(\Omega \times \mathbb{R})$-measurable and essentially bounded;

(ii) the set $D_{\varphi}=\{(x, z) \in \Omega \times \mathbb{R}: \varphi$ is discontinuous at $(x, z)\}$ belongs to $\mathcal{F}$

(iii) $\varphi^{-1}(r) \backslash \operatorname{int}\left(\varphi^{-1}(r)\right) \in \mathcal{F}$ for every $r \in \psi((\alpha, \beta))$;

(iv) $\overline{\varphi\left(S \backslash D_{\varphi}\right)} \subseteq \psi((\alpha, \beta))$.

Then, there exists $u \in W_{0}^{1, p}(\Omega)$ such that

$$
\psi\left(-\Delta_{p} u\right)=\varphi(x, u) \quad \text { in } \Omega .
$$

We finally point out that existence results for implicit equations involving such operators have been obtained with very different techniques by $[1,5,8,21]$.

\subsection{Structure of the Paper}

In Sect. 2 we will introduce the functional analytic setting we will use throughout the work. In Sect. 3 we will suppose $\varphi(x, \cdot, \cdot)$ to be continuous. Here we will consider some cases, according to the growth conditions on $\varphi$ or to the choice of the set $Y$. We will also give examples where these situations apply. In Sect. 4 we will consider the discontinuous framework. 


\section{Preliminaries}

Let $X$ be a topological space and let $V \subset X$. We denote by $\operatorname{int}(V)$ the interior of $V$ and by $\bar{V}$ the closure of $V$. The symbol $\mathcal{B}(X)$ is used to denote the Borel $\sigma$-algebra of $X$.

If $(X, d)$ is a metric space, for every $x \in X, r \geq 0$ and every nonempty set $V \subset X$, we define

$$
B(x, r)=\{z \in X: d(x, z) \leq r\} \quad \text { and } \quad d(x, V)=\inf _{z \in V} d(x, z) .
$$

Let $X$ and $Z$ be two nonempty sets. A multifunction $\Phi$ from $X$ into $Z$ (symbolically $\Phi: X \rightarrow 2^{Z}$ ) is a function from $X$ into the family of all subsets of $Z$. A function $\varphi: X \rightarrow Z$ is said to be a selection of $\Phi$ if $\varphi(x) \in \Phi(x)$ for all $x \in X$. For every set $W \subset Z$ we define $\Phi^{-}(W)=\{x \in X: \Phi(x) \cap W \neq \emptyset\}$.

Suppose that $(X, \mathcal{A})$ is a measurable space and $Z$ is a topological space. We say that the multifunction $\Phi$ is measurable if for every open set $W \subset Z$ we have $\Phi^{-}(W) \in \mathcal{A}$. Suppose now that $X$ and $Z$ are two topological spaces. We say that $\Phi$ is lower semicontinuous (resp. upper semicontinuous) if for every open (resp. closed) set $W \subset Z$ the set $\Phi^{-}(W)$ is open (resp. closed) in $X$. When $(Z, \delta)$ is a metric space, the multifunction $\Phi$ is lower semicontinuous if and only if, for every $z \in Z$, the real-valued function $x \mapsto \delta(z, \Phi(x))$, $x \in X$, is upper semicontinuous (see [20, Theorem 1.1]). If, moreover, $X$ is first countable, then $\Phi$ is lower semicontinuous if and only if, for every $x \in X$, every sequence $\left\{x_{k}\right\}$ in $X$ converging to $x$ and every $z \in \Phi(x)$, there exists a sequence $\left\{z_{k}\right\}$ in $Z$ converging to $z$ and such that $z_{k} \in \Phi\left(x_{k}\right)$, for all $k \in \mathbb{N}$ (see [10, Theorem 7.1.7]).

A general result on the lower semicontinuity of a multifunction is the following

Theorem 2.1 (Theorem 1.1 of [19]). Let $C, D$ be two topological spaces, with $D$ connected and locally connected, and let $f: C \times D \rightarrow \mathbb{R}$. For all $x \in C$ we set

$$
\begin{aligned}
V(x) & :=\{y \in D: f(x, y)=0\}, \\
M(x) & :=\{y \in D: y \text { is a local extremum point for } f(x, \cdot)\}, \\
\text { and } \quad & Q(x):=V(x) \backslash M(x) .
\end{aligned}
$$

Suppose that

(a) for all $x \in C, f(x, \cdot)$ is continuous, and $0 \in \operatorname{int}(f(x, D))$;

(b) for all $x \in C$ and for all $A$ open subset of $D$, there exists $\bar{y} \in A$ such that $f(x, \bar{y}) \neq 0$;

(c) the set

$$
\left\{\left(y^{\prime}, y^{\prime \prime}\right) \in D \times D:\left\{x \in C: f\left(x, y^{\prime}\right)<0<f\left(x, y^{\prime \prime}\right)\right\} \text { is open }\right\}
$$

is dense in $D \times D$.

Then, the multifunction $Q$ is lower semicontinuous, with nonempty closed values. 
From now on, $\Omega$ is a bounded domain in $\mathbb{R}^{N}, N \geq 2$, with a smooth boundary $\partial \Omega$. The symbol $\mathcal{L}(\Omega)$ denotes the Lebesgue $\sigma$-algebra of $\Omega$, while $m(\Omega)$ stands for the measure of $\Omega$.

Let $1 \leq r<\infty$. We denote by $L^{r}(\Omega), L^{r}\left(\Omega, \mathbb{R}^{N}\right)$, and $W^{1, r}(\Omega)$ the usual Lebesgue and Sobolev spaces equipped with the norms $\|\cdot\|_{r}$ and $\|\cdot\|_{1, r}$ given by

$$
\begin{aligned}
\|u\|_{r} & =\left(\int_{\Omega}|u|^{r} \mathrm{~d} x\right)^{1 / r}, \quad\|\nabla u\|_{r}=\left(\int_{\Omega}|\nabla u|^{r} \mathrm{~d} x\right)^{1 / r}, \\
\|u\|_{1, r} & =\left(\int_{\Omega}|u|^{r} \mathrm{~d} x\right)^{1 / r}+\left(\int_{\Omega}|\nabla u|^{r} \mathrm{~d} x\right)^{1 / r} .
\end{aligned}
$$

For $r=\infty$ we recall that the norm of $L^{\infty}(\Omega)$ is given by

$$
\|u\|_{\infty}=\operatorname{ess} \sup _{\Omega}|\mathrm{u}| \text {. }
$$

Furthermore, we denote by $W_{0}^{1, p}(\Omega)$ the closure of $C_{0}^{\infty}(\Omega)$ in $W^{1, p}(\Omega)$ and endow it with the norm

$$
\|u\|:=\left(\int_{\Omega}|\nabla u(x)|^{p} \mathrm{~d} x\right)^{1 / p}, \quad u \in W_{0}^{1, p}(\Omega) .
$$

It is well known that the Sobolev embedding theorem guarantees the existence of a linear, continuous map $i: W_{0}^{1, p}(\Omega) \rightarrow L^{p^{*}}(\Omega)$, with the critical exponent given by

$$
p^{*}= \begin{cases}\frac{N p}{N-p} & \text { if } p<N \\ +\infty & \text { otherwise }\end{cases}
$$

In particular, the embedding $W_{0}^{1, p}(\Omega) \hookrightarrow L^{r}(\Omega)$ is compact provided $1 \leq r<$ $p^{*}$.

If $p \neq N$, then to each $r \in\left[1, p^{*}\right]$ there corresponds a constant $c_{r p}>0$ satisfying

$$
\|u\|_{r} \leq c_{r p}\|u\|, \quad \forall u \in W_{0}^{1, p}(\Omega) .
$$

On the other hand, when $p=N$, for every $r \in[1, \infty)$ we have

$$
\|u\|_{r} \leq c_{r N}\|u\|, \quad \forall u \in W_{0}^{1, N}(\Omega) .
$$

When $p>N$, the embedding $W_{0}^{1, p}(\Omega) \hookrightarrow L^{\infty}(\Omega)$ implies the existence of a suitable $a>0$ such that

$$
\|u\|_{\infty} \leq a\|u\|, \quad \forall u \in W_{0}^{1, p}(\Omega),
$$

see $[3$, Ch. IX].

Given $p \in(1, \infty)$, the symbol $p^{\prime}$ denotes the conjugate exponent of $p$ while $W^{-1, p^{\prime}}(\Omega)$ stands for the dual space of $W^{1, p}(\Omega)$, with corresponding norm $\|\cdot\|_{-1, p^{\prime}}$. From $[3$, Theorem 6.4] we have the compact embedding $L^{p^{\prime}}(\Omega) \hookrightarrow W^{-1, p^{\prime}}(\Omega)$, and therefore there exists $b>0$ such that

$$
\|v\|_{-1, p^{\prime}} \leq b\|v\|_{p^{\prime}}, \quad \forall v \in L^{p^{\prime}}(\Omega) .
$$


Let $A_{p}: W_{0}^{1, p}(\Omega) \rightarrow W^{-1, p^{\prime}}(\Omega)$ be the nonlinear operator stemming from the negative $p$-Laplacian, that is

$$
\left\langle A_{p}(u), v\right\rangle:=\int_{\Omega}|\nabla u(x)|^{p-2} \nabla u(x) \cdot \nabla v(x) \mathrm{d} x, \quad u, v \in W_{0}^{1, p}(\Omega),
$$

and let $\lambda_{1, p}$ be its first eigenvalue in $W_{0}^{1, p}(\Omega)$. The following facts are well known (see, e.g., [18, Appendix A] or [11]):

$\left(\mathrm{p}_{1}\right) A_{p}$ is bijective and uniformly continuous on bounded sets;

$\left(\mathrm{p}_{2}\right)$ the inverse operator $A_{p}^{-1}$ is $\left(W^{-1, p^{\prime}}(\Omega), W_{0}^{1, p}(\Omega)\right)$-continuous;

$\left(\mathrm{p}_{3}\right)\left\|A_{p}(u)\right\|_{-1, p^{\prime}}=\|u\|_{p}^{p-1}$ in $W_{0}^{1, p}(\Omega)$;

$\left(\mathrm{p}_{4}\right)\|u\|_{p}^{p} \leq \frac{1}{\lambda_{1, p}}\|u\|^{p}$, for all $u \in W_{0}^{1, p}(\Omega)$.

\section{The Case When $\varphi$ is a Carathéodory Function}

In this section we consider the following problem: find $u \in W_{0}^{1, p}(\Omega)$ such that $\Delta_{p} u \in L^{p^{\prime}}(\Omega)$ and

$$
\psi\left(-\Delta_{p} u\right)=\varphi(x, u, \nabla u) .
$$

We first suppose that $Y=\mathbb{R}$ and state the following assumptions

(i) $\psi$ is non-constant on intervals;

(ii) for all $(x, z, w) \in \Omega \times \mathbb{R} \times \mathbb{R}^{N}$, the function $y \mapsto \varphi(x, z, w)-\psi(y)$ changes sign.

Theorem 3.1. Let $\varphi: \Omega \times \mathbb{R} \times \mathbb{R}^{N} \rightarrow \mathbb{R}$ be a Carathéodory function and let $\psi: \mathbb{R} \rightarrow \mathbb{R}$ be continuous. Suppose that (i)-(ii) hold true and, moreover,

(iii) there exist $a \in L^{p^{\prime}}\left(\Omega, \mathbb{R}_{0}^{+}\right), b, c \geq 0$, with $\frac{b}{\lambda_{1, p}}+\frac{c}{\lambda_{1, p}^{1 / p}}<1$, such that

$$
\sup \left\{|y|: y \in \psi^{-1}(\varphi(x, z, w))\right\}<a(x)+b|z|^{p-1}+c|w|^{p-1}
$$

for all $(x, z, w) \in \Omega \times \mathbb{R} \times \mathbb{R}^{N}$.

Then, there exists a solution $u \in W_{0}^{1, p}(\Omega)$ to Eq. (3.1).

Proof. Fix $x \in \Omega$. We want to apply Theorem 2.1. To this end, we choose $C=\mathbb{R} \times \mathbb{R}^{N}, D=\mathbb{R}, f(z, w, y)=\varphi(x, z, w)-\psi(y)$, and for every $(z, w) \in$ $\mathbb{R} \times \mathbb{R}^{N}$ we set

$$
\begin{aligned}
F(x, z, w):=\{y \in \mathbb{R}: \varphi(x, z, w)-\psi(y)=0, \\
y \text { is not a local extremum point of } \psi(\cdot)\} .
\end{aligned}
$$

Hypothesis (ii) directly yields (a). Moreover, in order to verify (b), we need to check that for all $(z, w) \in \mathbb{R} \times \mathbb{R}^{N}$ the set $U:=\{y \in \mathbb{R}: \varphi(x, z, w)-\psi(y) \neq 0\}$ is dense in $\mathbb{R}$. Assumption (i) implies that $\mathbb{R} \backslash U$ has empty interior, therefore $U$ is dense in $\mathbb{R}$, as desired.

Let us next consider the set

$$
\begin{aligned}
\mathcal{A}:= & \left\{\left(y^{\prime}, y^{\prime \prime}\right) \in \mathbb{R} \times \mathbb{R}:\left\{(z, w) \in \mathbb{R} \times \mathbb{R}^{N}: \varphi(x, z, w)-\psi\left(y^{\prime}\right)<0\right.\right. \\
& \left.\left.<\varphi(x, z, w)-\psi\left(y^{\prime \prime}\right)\right\} \text { is open }\right\} .
\end{aligned}
$$


We want to show that $\mathcal{A}$ is dense in $\mathbb{R} \times \mathbb{R}$. Suppose that there exist $y^{\prime}, y^{\prime \prime} \in \mathbb{R}$ such that

$$
\varphi(x, z, w)-\psi\left(y^{\prime}\right)<0<\varphi(x, z, w)-\psi\left(y^{\prime \prime}\right),
$$

that is, $\varphi(x, z, w) \in\left(\psi\left(y^{\prime \prime}\right), \psi\left(y^{\prime}\right)\right)$. Then the continuity of the function $\varphi(x, \cdot, \cdot)$ implies that the set

$$
B:=\left\{(z, w) \in \mathbb{R} \times \mathbb{R}^{N}: \varphi(x, z, w)-\psi\left(y^{\prime}\right)<0<\varphi(x, z, w)-\psi\left(y^{\prime \prime}\right)\right\},
$$

is open. If it is not possible to find such $y^{\prime}, y^{\prime \prime}$ that realize (3.2), then the set $B$ is empty. This implies that $\mathcal{A}=\mathbb{R} \times \mathbb{R}$, and then (c) follows.

Thanks to Theorem 2.1, the multifunction $F(x, \cdot, \cdot)$ is lower semicontinuous, with nonempty closed values.

Moreover, thanks to [6, Lemma III.14], for all $y^{\prime}, y^{\prime \prime} \in \mathbb{R}$ we have

$$
\begin{aligned}
& \left\{(x, z, w) \in \Omega \times \mathbb{R} \times \mathbb{R}^{N}: \varphi(x, z, w)-\psi\left(y^{\prime}\right)<0<\varphi(x, z, w)-\psi\left(y^{\prime \prime}\right)\right\} \\
& \quad=\left\{(x, z, w) \in \Omega \times \mathbb{R} \times \mathbb{R}^{N}: \varphi(x, z, w) \in\left(\psi\left(y^{\prime \prime}\right), \psi\left(y^{\prime}\right)\right)\right\} \\
& \quad \in \mathcal{L}(\Omega) \otimes \mathcal{B}\left(\mathbb{R} \times \mathbb{R}^{N}\right) .
\end{aligned}
$$

Therefore, setting $\Lambda^{*}=\mathbb{R} \times \mathbb{R}$ we see that condition (iii) of [13, Theorem 3.2] is satisfied. Fix now an open set $A \subset \mathbb{R}$. Arguing again as in [13, Theorem 3.2] we see that

$$
\begin{aligned}
F^{-}(A)= & \bigcup_{\left(y^{\prime}, y^{\prime \prime}\right) \in A \times A}\left\{(x, z, w) \in \Omega \times \mathbb{R} \times \mathbb{R}^{N}:\right. \\
& \left.\varphi(x, z, w)-\psi\left(y^{\prime}\right)<0<\varphi(x, z, w)-\psi\left(y^{\prime \prime}\right)\right\} .
\end{aligned}
$$

Then (3.3) implies that $F^{-}(A) \in \mathcal{L}(\Omega) \otimes \mathcal{B}\left(\mathbb{R} \times \mathbb{R}^{N}\right)$ and therefore $F$ is measurable.

Finally, fix any $y \in F(x, z, w)$. By hypothesis (iii) we have

$$
\inf _{y \in F(x, z, w)}|y|<a(x)+b|z|^{p-1}+c|w|^{p-1} \quad \text { in } \Omega \times \mathbb{R} \times \mathbb{R}^{N} .
$$

Therefore, all the hypotheses of Theorem 1.1 are satisfied, and there exists $u \in W_{0}^{1, p}(\Omega)$ such that $-\Delta_{p} u=F(x, u, \nabla u)$. By definition of $F$ we then have the result.

Remark 3.2. We now discuss a very simple situation when hypothesis (iii) applies.

Suppose that $\varphi\left(\Omega \times \mathbb{R} \times \mathbb{R}^{N}\right) \subset[\alpha, \beta]$ and $\psi$ is such that $\psi^{-1}(B)$ is bounded, for every bounded $B \subset \mathbb{R}$. If $(x, z, w) \in \Omega \times \mathbb{R} \times \mathbb{R}^{N}$, we get $\varphi(x, z, w) \in[\alpha, \beta]$, and so $\psi^{-1}(\varphi(x, z, w)) \subset \psi^{-1}([\alpha, \beta])$. Then, if we choose $a \in L^{p^{\prime}}\left(\Omega, \mathbb{R}_{0}^{+}\right)$such that $a(x)>\sup \left\{|y|: y \in \psi^{-1}([\alpha, \beta])\right\}$ for all $x \in \Omega$, we have

$$
\left|\psi^{-1}(\varphi(x, z, w))\right|<a(x) \text { in } \Omega \times \mathbb{R} \times \mathbb{R}^{N},
$$

that is hypothesis (iii) with $b=c=0$.

As an application of the previous result, we consider the following 
Corollary 3.3. Let $g \in L^{2}(\Omega)$ and $\gamma \in(0,1)$. Then, for every $\lambda \neq 0$ and $\mu \in \mathbb{R}$ there exists a solution $u \in W_{0}^{1,2}(\Omega)$ to the equation

$$
-\Delta u=g(x)+\mu(|u|+|\nabla u|)^{\gamma}+\lambda \sin (-\Delta u) .
$$

Proof. Fix $\lambda \neq 0$ and $\mu \in \mathbb{R}$. For every $(x, z, w) \in \Omega \times \mathbb{R} \times \mathbb{R}^{N}$ and every $y \in \mathbb{R}$ we set

$$
\varphi(x, z, w):=g(x)+\mu(|z|+|w|)^{\gamma} \quad \text { as well as } \quad \psi(y):=y-\lambda \sin y .
$$

Since $\lim _{y \rightarrow \pm \infty}(y-\lambda \sin y)= \pm \infty$, the function $y \mapsto \varphi(x, z, w)-\psi(y)$ changes sign, and then hypothesis (ii) follows. Moreover, $\psi$ vanishes only at points of $\mathbb{R}$ and not in intervals, which implies that also hypothesis (i) is satisfied.

Fix now $(x, z, w) \in \Omega \times \mathbb{R} \times \mathbb{R}^{N}$. In order to verify hypothesis (iii), we want to find $b, c \geq 0$, with $\frac{b}{\lambda_{1,2}}+\frac{c}{\lambda_{1,2}^{1 / 2}}<1$, and $a \in L^{2}\left(\Omega, \mathbb{R}_{0}^{+}\right)$such that

$$
\max \left\{|y|: y \in \psi^{-1}(\varphi(x, z, w))\right\}<a(x)+b|z|+c|w|,
$$

or equivalently $|y|<a(x)+b|z|+c|w|$ for every $y$ solution to the equation

$$
\psi(y)=\varphi(x, z, w) .
$$

We point out that in (3.5) the maximum replaces the supremum because the set $\psi^{-1}(\varphi(x, z, w))$ is compact. Let $\tilde{y}$ be a solution to (3.6). Then Young's inequality with exponents $1 / \gamma$ and $1 /(1-\gamma)$ gives

$$
\begin{aligned}
|\psi(\tilde{y})|=|\varphi(x, z, w)| & =\left|g(x)+\mu(|z|+|w|)^{\gamma}\right| \\
& \leq|g(x)|+|\mu||z|^{\gamma}+|\mu||w|^{\gamma} \\
& \leq|g(x)|+\varepsilon|z|+\varepsilon|w|+C_{\gamma, \varepsilon, \mu} \\
& \leq \tilde{g}(x)+\varepsilon|z|+\varepsilon|w|,
\end{aligned}
$$

where $\tilde{g}(x):=|g(x)|+C_{\gamma, \varepsilon, \mu}$ for every $x \in \Omega$. On the other hand, by the definition of $\psi$ we have

$$
|\psi(\tilde{y})|=|\tilde{y}-\lambda \sin \tilde{y}| \geq|\tilde{y}|-|\lambda|,
$$

and then (3.7) gives

$$
\begin{aligned}
|\tilde{y}| & \leq|\psi(\tilde{y})|+|\lambda| \\
& \leq \tilde{g}(x)+|\lambda|+\varepsilon|z|+\varepsilon|w| \\
& <\bar{g}(x)+\varepsilon|z|+\varepsilon|w|,
\end{aligned}
$$

where $\bar{g}(x):=\tilde{g}(x)+2|\lambda|$, for every $x \in \Omega$. Observe that $\bar{g} \in L^{2}\left(\Omega, \mathbb{R}_{0}^{+}\right)$. If we choose $\varepsilon$ in such a way that

$$
\frac{\varepsilon}{\lambda_{1,2}}+\frac{\varepsilon}{\lambda_{1,2}^{1 / 2}}<1
$$

then hypothesis (iii) is satisfied with $a:=\bar{g}$ and $b:=c:=\varepsilon$. Thanks to Theorem 3.1, there exists a solution $u \in W_{0}^{1,2}(\Omega)$ to Eq. (3.4).

In the following situation the function $\psi$ exhibits a very different behavior. 
Corollary 3.4. Let $p \in[2,+\infty), f \in L^{p^{\prime}}(\Omega)$, and $\gamma \in(0, p-1)$. Then, for every $\mu \in \mathbb{R}$ and $\lambda>0$, there exists a solution $u \in W_{0}^{1, p}(\Omega)$ to the equation

$$
-\Delta_{p} u=f(x)+\mu(|u|+|\nabla u|)^{\gamma}-\lambda e^{-\Delta_{p} u} .
$$

Proof. Fix $\mu \in \mathbb{R}$ and $\lambda>0$. As before, for every $(x, z, w) \in \Omega \times \mathbb{R} \times \mathbb{R}^{N}$ and every $y \in \mathbb{R}$ we set

$$
\varphi(x, z, w):=f(x)+\mu(|z|+|w|)^{\gamma} \text { as well as } \psi(y):=y+\lambda e^{y} .
$$

Since $\lim _{y \rightarrow \pm \infty}\left(y+\lambda e^{y}\right)= \pm \infty$, then hypotheses (i) and (ii) are fulfilled. In order to verify hypothesis (iii), we argue as in Corollary 3.3. Let $\tilde{y}$ be a solution to $\varphi(x, z, w)-\psi(y)=0$, then Young's inequality with exponents $\frac{p-1}{\gamma}, \frac{p-1}{p-1-\gamma}>1$ gives

$$
\begin{aligned}
|\psi(\tilde{y})|=|\varphi(x, z, w)| & =\left|f(x)+\mu(|z|+|w|)^{\gamma}\right| \\
& \leq|f(x)|+2^{\gamma}\left(|\mu||z|^{\gamma}+|\mu||w|^{\gamma}\right) \\
& \leq|f(x)|+\varepsilon|z|^{p-1}+\varepsilon|w|^{p-1}+C_{\gamma, \varepsilon, \mu} \\
& =\tilde{f}(x)+\varepsilon|z|^{p-1}+\varepsilon|w|^{p-1},
\end{aligned}
$$

where $\tilde{f}(x):=|f(x)|+C_{\gamma, \varepsilon, \mu}$ for every $x \in \Omega$.

On the other hand we have

$$
|\psi(\tilde{y})|=\left|\tilde{y}+\lambda e^{\tilde{y}}\right| \geq|\tilde{y}|-|\xi|,
$$

$\xi \not \equiv 0$ being the unique solution to the equation $y+\lambda e^{y}=0$. Let us show (3.9) for a general $y \in \mathbb{R}$. If $y \geq \xi$ we have

$$
\begin{aligned}
\left|y+\lambda e^{y}\right| & =\left|y+\lambda e^{y}-\xi-\lambda e^{\xi}\right| \\
& =\left|y-\xi+\lambda\left(e^{y}-e^{\xi}\right)\right| \\
& \geq|y-\xi| \geq|y|-|\xi| .
\end{aligned}
$$

Suppose now that $y<\xi$, then

$$
\begin{aligned}
\left|y+\lambda e^{y}\right| & =\left|y-\xi+\lambda\left(e^{y}-e^{\xi}\right)\right| \\
& =\left|\xi-y+\lambda\left(e^{\xi}-e^{y}\right)\right| \\
& \geq|\xi-y| \geq|y|-|\xi| .
\end{aligned}
$$

From (3.9) we then have

$$
\begin{aligned}
|\tilde{y}| & \leq|\psi(\tilde{y})|+|\xi| \\
& \leq \tilde{f}(x)+\varepsilon|z|^{p-1}+\varepsilon|w|^{p-1}+|\xi| \\
& <\bar{f}(x)+\varepsilon|z|^{p-1}+\varepsilon|w|^{p-1},
\end{aligned}
$$

with $\bar{f}(x):=\tilde{f}(x)+2|\xi|$ for every $x \in \Omega$. Observe that $\bar{f} \in L^{p^{\prime}}\left(\Omega, \mathbb{R}_{0}^{+}\right)$. Then, if we choose $\varepsilon$ in such a way that

$$
\frac{\varepsilon}{\lambda_{1, p}}+\frac{\varepsilon}{\lambda_{1, p}^{1 / p}}<1,
$$

hypothesis (iii) is satisfied with $a:=\bar{f}$ and $b:=c:=\varepsilon$. Therefore, Theorem 3.1 gives the existence of a solution $u \in W_{0}^{1, p}(\Omega)$ to Eq. (3.8). 
In order to state our next theorem, we need some preliminary results. The following is an a priori estimate on $\|\nabla u\|_{L^{\infty}\left(\Omega ; \mathbb{R}^{N}\right)}$, see $[17$, Proposition 3.3] or [7, Theorem 1.3].

Proposition 3.5. Suppose $q>N$. Then, there exists a constant $\hat{C}>0$, depending on $p, q$, and $\Omega$, such that

$$
\|\nabla u\|_{L^{\infty}\left(\Omega, \mathbb{R}^{N}\right)} \leq \hat{C}\left\|\Delta_{p} u\right\|_{L^{q}(\Omega)}^{1 /(p-1)}
$$

Proposition 3.5 is used in the proof of the following

Theorem 3.6. Let $p \in(1, \infty), q>N$, and let $F: \Omega \times \mathbb{R} \times \mathbb{R}^{N} \rightarrow 2^{\mathbb{R}}$ be a closed-valued multifunction. Suppose that

$\left(\mathrm{h}_{1}\right) \quad F$ is $\mathcal{L}(\Omega) \otimes \mathcal{B}\left(\mathbb{R} \times \mathbb{R}^{N}\right)$-measurable;

$\left(\mathrm{h}_{2}\right)$ for almost every $x \in \Omega$ the multifunction $(z, w) \mapsto F(x, z, w)$ turns out to be lower semicontinuous;

$\left(\mathrm{h}_{3}\right)$ for appropriate $a \in L^{q}\left(\Omega, \mathbb{R}_{0}^{+}\right)$and $\xi: \mathbb{R}_{0}^{+} \times \mathbb{R}_{0}^{+} \rightarrow \mathbb{R}_{0}^{+}$nondecreasing with respect to each variable separately one has

$$
\inf _{y \in F(x, z, w)}|y|<a(x)+\xi(|z|,|w|) \quad \text { in } \Omega \times \mathbb{R} \times \mathbb{R}^{N} ;
$$

$\left(\mathrm{h}_{4}\right)$ there exists $R>0$ such that

$$
\|a\|_{q}+m(\Omega)^{1 / q} \xi\left(\delta_{\Omega} \hat{C} R^{1 /(p-1)}, \hat{C} R^{1 /(p-1)}\right) \leq R,
$$

where $\delta_{\Omega}:=\operatorname{diam}(\Omega)$ and $\hat{C}$ is given by Proposition 3.5.

Then, there exists at least one solution $u \in W_{0}^{1, p}(\Omega)$ to problem

$$
\begin{aligned}
-\Delta_{p} u & \in F(x, u, \nabla u) & & \text { in } \Omega, \\
u & =0 & & \text { on } \partial \Omega .
\end{aligned}
$$

Finally, we state our result.

Theorem 3.7. Let $\varphi$ and $\psi$ as in Theorem 3.1. Suppose that hypotheses (i)-(ii) hold true and, moreover,

(iii) $)^{\prime}$ there exist $a \in L^{q}\left(\Omega, \mathbb{R}_{0}^{+}\right), q>N, g: \mathbb{R}_{0}^{+} \times \mathbb{R}_{0}^{+} \rightarrow \mathbb{R}_{0}^{+}$nondecreasing with respect to each variable separately, such that

$$
\sup \left\{|y|: y \in \psi^{-1}(\varphi(x, z, w))\right\}<a(x)+g(|z|,|w|),
$$

for all $(x, z, w) \in \Omega \times \mathbb{R} \times \mathbb{R}^{N}$;

(iv) there exists $R>0$ such that

$$
\|a\|_{L^{q}(\Omega)}+m(\Omega)^{1 / q} g\left(\delta_{\Omega} \hat{C} R^{1 /(p-1)}, \hat{C} R^{1 /(p-1)}\right) \leq R,
$$

where $\hat{C}$ comes from Proposition 3.5.

Then, Eq. (3.1) has a solution $u \in W_{0}^{1, p}(\Omega)$.

Proof. We aim to apply Theorem 3.6. As before, fix $x \in \Omega$ and for all $(z, w) \in$ $\mathbb{R} \times \mathbb{R}^{N}$ define

$$
F(x, z, w):=\{y \in \mathbb{R}: \varphi(x, z, w)-\psi(y)=0,
$$

$y$ is not a local extremum point of $\psi(\cdot)\}$. 
Reasoning as in Theorem 3.1 ensures that $F$ has nonempty closed values, is lower semicontinuous w.r.t. $(z, w)$, and $\mathcal{L}(\Omega) \otimes \mathcal{B}\left(\mathbb{R} \times \mathbb{R}^{N}\right)$-measurable.

Fix now $y \in F(x, z, w)$, that is $y \in \psi^{-1}(\varphi(x, z, w))$. Then hypothesis (iii)' implies that

$$
\inf _{y \in F(x, z, w)}|y|<a(x)+g(|z|,|w|) \quad \text { in } \Omega \times \mathbb{R} \times \mathbb{R}^{N} .
$$

Taking into account (iv), we see that all the hypotheses of Theorem 3.6 are fulfilled. Therefore, there exists $u \in W_{0}^{1, p}(\Omega)$ such that $-\Delta_{p} u \in F(x, u, \nabla u)$. According to the definition of $F$, it turns out that $u$ is a solution to Eq. (3.1).

The following result is an application of the previous theorem and has been inspired by [9, Corollary 1]. Observe that, unlike [9], here we consider a function $\varphi$ which is not necessarily continuous w.r.t. the variable $x$, but only lies in a suitable $L^{q}(\Omega)$. Moreover, here we deal with partial differential equations.

Corollary 3.8. Let $h \in L^{q}(\Omega)$, with $q>N$. Then, for every $k \neq 0$ and every sufficiently small $\|h\|_{q}$ there exists a solution $u \in W_{0}^{1,2}(\Omega)$ to the equation

$$
-\Delta u=h(x)+u^{3}+|\nabla u|^{2}+k \sin (-\Delta u) .
$$

Proof. Fix $k \in \mathbb{R}$ and for all $(x, z, w) \in \Omega \times \mathbb{R} \times \mathbb{R}^{N}$ and all $y \in \mathbb{R}$ define

$$
\varphi(x, z, w):=h(x)+z^{3}+|w|^{2} \quad \text { as well as } \quad \psi(y):=y-k \sin y .
$$

Reasoning like in Corollary 3.3 gives that hypotheses (i)-(ii) are fulfilled.

In order to verify hypothesis (iii) $)^{\prime}$, let $g(|z|,|w|):=|z|^{3}+|w|^{2}$ for all $(z, w) \in \mathbb{R} \times \mathbb{R}^{N}$. It turns out that $g: \mathbb{R}_{0}^{+} \times \mathbb{R}_{0}^{+} \rightarrow \mathbb{R}_{0}^{+}$is nondecreasing w.r.t. each variable, separately. Let $\tilde{y}$ be a solution to the equation $\psi(y)=$ $\varphi(x, z, w)$. It follows that

$$
\begin{aligned}
|\psi(\tilde{y})| & =|\varphi(x, z, w)| \\
& \leq|h(x)|+|z|^{3}+|w|^{2} \\
& =|h(x)|+g(|z|,|w|) .
\end{aligned}
$$

On the other hand, since $|\psi(\tilde{y})|=|\tilde{y}-k \sin \tilde{y}| \geq|\tilde{y}|-|k|$, then we have

$$
\begin{aligned}
|\tilde{y}| & \leq|\psi(\tilde{y})|+|k| \\
& \leq|h(x)|+g(|z|,|w|)+|k| \\
& <\bar{h}(x)+g(|z|,|w|),
\end{aligned}
$$

where $\bar{h}(x):=|h(x)|+2|k|$ for every $x \in \Omega$ and $\bar{h} \in L^{q}\left(\Omega, \mathbb{R}_{0}^{+}\right)$. Hence hypothesis (iii)' follows.

In order to verify hypothesis (iv), we have to check the existence of $R>0$ such that

$$
\|\bar{h}\|_{L^{q}(\Omega)}+m(\Omega)^{1 / q} \delta_{\Omega}^{3} \hat{C}^{3} R^{3}+m(\Omega)^{1 / q} \hat{C}^{2} R^{2} \leq R .
$$

If $0<R<<1$, then choosing $\bar{h}$ in such a way that $\|\bar{h}\|_{L^{q}(\Omega)}<\frac{R}{2}$ gives immediately (3.10), since the terms containing $R^{2}$ and $R^{3}$ are negligible with respect to $R$. Therefore, all the hypotheses of Theorem 3.7 are fulfilled, and we have the thesis. 
The next result provides solutions to Eq. (3.1) when the function $\psi$ is of the form $y \mapsto y-h(y)$, with $h$ continuous and bounded. Note that here a specific growth condition on $\varphi$ is required.

Theorem 3.9. Let $\varphi: \Omega \times \mathbb{R} \times \mathbb{R}^{N} \rightarrow \mathbb{R}$ be a Carathéodory function and let $h \in L^{\infty}(\mathbb{R})$ be continuous. Suppose that (i)-(ii) hold true and, moreover, (iii)" there exist $f \in L^{p^{\prime}}\left(\Omega, \mathbb{R}_{0}^{+}\right)$, with $f(x) \geq\|h\|_{\infty}$ for all $x \in \Omega, \mu>0$, and $\gamma \in(0, p-1)$ such that

$$
\sup _{(x, z, w) \in \Omega \times \mathbb{R} \times \mathbb{R}^{N}}|\varphi(x, z, w)|<f(x)+\mu(|z|+|w|)^{\gamma} .
$$

Then, there exists a solution $u \in W_{0}^{1, p}(\Omega)$ to the equation

$$
-\Delta_{p} u-h\left(-\Delta_{p} u\right)=\varphi(x, u, \nabla u) .
$$

Proof. We fix $x \in \Omega$ and for all $(z, w) \in \mathbb{R} \times \mathbb{R}^{N}$ define

$$
\begin{aligned}
F(x, z, w):=\{y \in \mathbb{R}: & \varphi(x, z, w)-(y-h(y))=0, \\
& y \text { is not a local extremum point of } y \mapsto y-h(y)\} .
\end{aligned}
$$

Reasoning as in the above proofs ensures that $F$ is lower semicontinuous w.r.t. $(z, w), \mathcal{L}(\Omega) \otimes \mathcal{B}\left(\mathbb{R} \times \mathbb{R}^{N}\right)$-measurable, and has nonempty, closed values.

Fix $(x, z, w) \in \Omega \times \mathbb{R} \times \mathbb{R}^{N}$. If $y \in F(x, z, w)$, then it solves the equation $\varphi(x, z, w)=y-h(y)$. We first suppose that $\gamma \in[1, p-1)$. Then Young's inequality with exponents $\frac{p-1}{\gamma}, \frac{p-1}{p-1-\gamma}>1$ gives

$$
\begin{aligned}
|y| & \leq|y-h(y)|+|h(y)| \\
& \leq|\varphi(x, z, w)|+\|h\|_{\infty} \\
& <f(x)+\mu(|z|+|w|)^{\gamma}+\|h\|_{\infty} \\
& \leq 2 f(x)+2^{\gamma-1} \mu\left(|z|^{\gamma}+|w|^{\gamma}\right) \\
& \leq 2 f(x)+2^{\gamma-1} \mu\left(\varepsilon|z|^{p-1}+\varepsilon|w|^{p-1}+K_{\varepsilon}\right) \\
& \leq 2 f(x)+C_{\varepsilon}+2^{\gamma-1} \mu \varepsilon\left(|z|^{p-1}+|w|^{p-1}\right),
\end{aligned}
$$

where $C_{\varepsilon}:=2^{\gamma-1} \mu K_{\varepsilon}$. Hence

$$
\inf _{y \in F(x, z, w)}|y|<2 f(x)+C_{\varepsilon}+2^{\gamma-1} \mu \varepsilon\left(|z|^{p-1}+|w|^{p-1}\right) .
$$

If we choose $\varepsilon$ in such a way that

$$
\frac{2^{\gamma-1} \mu \varepsilon}{\lambda_{1, p}}+\frac{2^{\gamma-1} \mu \varepsilon}{\lambda_{1, p}^{1 / p}}<1,
$$

hypothesis (h3) of Theorem 1.1 is fulfilled with $a:=2 f+C_{\varepsilon} \in L^{p^{\prime}}\left(\Omega, \mathbb{R}_{0}^{+}\right)$ and $b:=c:=2^{\gamma-1} \mu \varepsilon$.

Suppose now $\gamma \in(0,1)$. Since $(a+b)^{\gamma} \leq a^{\gamma}+b^{\gamma}$ for every $a, b \geq 0$, reasoning as before yields

$$
|y|<2 f(x)+\tilde{C}_{\varepsilon}+\mu \varepsilon\left(|z|^{p-1}+|w|^{p-1}\right),
$$

where $\tilde{C}_{\varepsilon}:=\mu K_{\varepsilon}$. If we now choose $\varepsilon$ in such a way that

$$
\frac{\mu \varepsilon}{\lambda_{1, p}}+\frac{\mu \varepsilon}{\lambda_{1, p}^{1 / p}}<1,
$$


hypothesis (h3) of Theorem 1.1 is again fulfilled with $a:=2 f+\tilde{C}_{\varepsilon} \in$ $L^{p^{\prime}}\left(\Omega, \mathbb{R}_{0}^{+}\right)$and $b:=c:=\mu \varepsilon$.

In both cases, there exists $u \in W_{0}^{1, p}(\Omega)$ such that $-\Delta_{p} u \in F(x, u, \nabla u)$, which gives a solution to Eq. (3.11).

We conclude this section considering the case when $Y$ is a closed interval of $\mathbb{R}$. Observe that here no growth conditions on $\varphi$ are required.

Theorem 3.10. Let $\varphi: \Omega \times \mathbb{R} \times \mathbb{R}^{N} \rightarrow \mathbb{R}$ be a Carathéodory function and let $\psi:[\alpha, \beta] \rightarrow \mathbb{R}$ be continuous. Suppose that

(1) $\psi$ is non-constant on intervals;

(2) for every $(x, z, w) \in \Omega \times \mathbb{R} \times \mathbb{R}^{N}$, the function $y \mapsto \varphi(x, z, w)-\psi(y)$ changes sign in $[\alpha, \beta]$.

Then, there exists a solution $u \in W_{0}^{1, p}(\Omega)$ to Eq. (3.1).

Proof. As before, fix $x \in \Omega$ and for all $(z, w) \in \mathbb{R} \times \mathbb{R}^{N}$ define

$$
F(x, z, w):=\{y \in[\alpha, \beta]: \varphi(x, z, w)-\psi(y)=0,
$$

$y$ is not a local extremum point of $\psi(\cdot)\}$.

A familiar argument ensures that $F$ takes nonempty closed values, is lower semicontinuous w.r.t. $(z, w)$ and $\mathcal{L}(\Omega) \otimes \mathcal{B}\left(\mathbb{R} \times \mathbb{R}^{N}\right)$-measurable.

If now $y \in F(x, z, w)$, then $|y| \leq \max \{|\alpha|,|\beta|\}$, and so hypothesis (h3) of Theorem 1.1 is immediately satisfied with $a(x):=2 \max \{|\alpha|,|\beta|\}$ for every $x \in \Omega$ and $b:=c:=0$. Therefore, there exists $u \in W_{0}^{1, p}(\Omega)$ such that $-\Delta_{p} u \in F(x, u, \nabla u)$, i.e., $u$ is a solution to (3.1).

We now consider two applications of the previous result, which differ by the behavior of the function $\psi$. In both cases, the boundedness of $\varphi$ will play a central role.

Corollary 3.11. Let $f \in L^{\infty}(\Omega), k \in \mathbb{N}, k$ even and such that $k \pi>\|f\|_{\infty}$, and let $\psi:[-k \pi, k \pi] \rightarrow \mathbb{R}$ be defined by $\psi(y)=y \cos y$. Then, there exists a solution $u \in W_{0}^{1, p}(\Omega)$ to the equation

$$
\psi\left(-\Delta_{p} u\right)=f(x) \text { in } \Omega .
$$

Proof. Assumption (1) is clearly satisfied. Moreover, for every $x \in \Omega$, we have

$$
f(x)-\psi(k \pi)=f(x)-k \pi \cos (k \pi)=f(x)-k \pi(-1)^{k}=f(x)-k \pi<0
$$

and $f(x)-\psi(-k \pi)=f(x)+k \pi \cos (-k \pi)=f(x)+k \pi>0$,

which gives hypothesis (2). Thanks to Theorem 3.10, there exists at least a solution $u \in W_{0}^{1, p}(\Omega)$ to Eq. (3.12).

Note that the interval $[\alpha, \beta]$ could be unbounded, as the following example shows.

Corollary 3.12. Let $p \in(1, \infty), f \in L^{p^{\prime}}(\Omega)$, and $\varphi: \Omega \times \mathbb{R} \times \mathbb{R}^{N} \rightarrow \mathbb{R}$. Suppose that there exists $\lambda>0$ such that

$$
\sup _{(x, z, w) \in \Omega \times \mathbb{R} \times \mathbb{R}^{N}}|\varphi(x, z, w)|<\lambda .
$$


Then, there exists a solution $u \in W_{0}^{1, p}(\Omega)$ to the equation

$$
\varphi(x, u, \nabla u)-\lambda e^{\Delta_{p} u}+\Delta_{p} u=0 .
$$

Proof. Define $\psi(y):=\lambda e^{-y}-y$ for every $y \in[0,+\infty)$. Observe that hypothesis (1) is immediately satisfied. Moreover, thanks to (3.13), for every $(x, z, w) \in \Omega \times \mathbb{R} \times \mathbb{R}^{N}$ we have

$$
\begin{aligned}
& \varphi(x, z, w)-\psi(0)=\varphi(x, z, w)-\lambda<0 \\
& \text { and } \lim _{y \rightarrow+\infty}(\varphi(x, z, w)-\psi(y))=+\infty,
\end{aligned}
$$

that is hypothesis (2), and hence the conclusion follows from Theorem 3.10.

\section{The Discontinuous Framework}

This section is devoted to the proof of Theorem 1.4, which we rewrite here, for the reader's convenience. Given $(x, z) \in S:=\Omega \times \mathbb{R}$, set $\pi_{0}(x, z)=x$ and $\pi_{1}(x, z)=z$. Moreover, fix $p>N$ and define

$$
\begin{aligned}
& \mathcal{F}=\{A \subset S: A \text { is measurable and there exists } i \in\{0,1\} \text { such that } \\
& \left.m\left(\pi_{i}(A)\right)=0\right\} .
\end{aligned}
$$

Theorem 4.1. Let $(\alpha, \beta) \subset \mathbb{R}$ be such that $0 \notin(\alpha, \beta)$, let $\psi:(\alpha, \beta) \rightarrow \mathbb{R}$ be continuous, and $\varphi: \Omega \times \mathbb{R} \rightarrow \mathbb{R}$. Suppose that

(i) $\varphi$ is $\mathcal{L}(\Omega \times \mathbb{R})$-measurable and essentially bounded;

(ii) the set $D_{\varphi}=\{(x, z) \in S: \varphi$ is discontinuous at $(x, z)\}$ belongs to $\mathcal{F}$;

(iii) $\varphi^{-1}(r) \backslash \operatorname{int}\left(\varphi^{-1}(r)\right) \in \mathcal{F}$ for every $r \in \psi((\alpha, \beta))$;

(iv) $\overline{\varphi\left(S \backslash D_{\varphi}\right)} \subset \psi((\alpha, \beta))$.

Then, there exists $u \in W_{0}^{1, p}(\Omega)$ such that

$$
\psi\left(-\Delta_{p} u\right)=\varphi(x, u) \quad \text { in } \Omega .
$$

Proof. The first part essentially follows the proof of [16, Theorem 3.1]. Thanks to assumption (i), there exists a constant $c>0$ such that

$$
S \backslash D_{\varphi} \subset\{(x, z) \in S:|\varphi(x, z)| \leq c\} .
$$

Set

$$
\hat{a}:=\min \overline{\varphi\left(S \backslash D_{\varphi}\right)} \quad \text { and } \quad \hat{b}:=\max \overline{\varphi\left(S \backslash D_{\varphi}\right)} .
$$

Thanks to hypothesis (iv) there exist $y^{\prime}, y^{\prime \prime} \in(\alpha, \beta)$ such that $\psi\left(y^{\prime}\right)=\hat{a}$ and $\psi\left(y^{\prime \prime}\right)=\hat{b}$. Let $\lambda:[0,1] \rightarrow(\alpha, \beta)$ be a continuous function such that $\lambda(0)=y^{\prime}, \lambda(1)=y^{\prime \prime}$. Moreover, let $\tilde{\psi}:[0,1] \rightarrow \mathbb{R}$ be defined by

$$
\tilde{\psi}(t):=\psi(\lambda(t)), \quad t \in[0,1] .
$$

We distinguish among two cases.

Suppose that $\tilde{\psi}$ is constant. Then $\hat{a}=\hat{b}$ and consequently $\varphi\left(S \backslash D_{\varphi}\right)=$ $\{\hat{a}\}$. Let $u \in W_{0}^{1, p}(\Omega)$ be such that $-\Delta_{p} u=y^{\prime}$. Since $\psi\left(-\Delta_{p} u\right)=\psi\left(y^{\prime}\right)=\hat{a}$, the conclusion will be achieved by showing that the set

$$
\Omega_{\varphi}:=\left\{x \in \Omega:(x, u(x)) \in D_{\varphi}\right\}
$$


has measure zero.

First of all observe that an elementary computation gives

$$
\Omega_{\varphi} \subset \pi_{0}\left(D_{\varphi}\right) \cap u^{-1}\left(\pi_{1}\left(D_{\varphi}\right)\right)
$$

and, due to (ii), $m\left(\pi_{i}\left(D_{\varphi}\right)\right)=0$ for some $i \in\{0,1\}$. Suppose $i=0$. From (4.2) we obtain

$$
m\left(\Omega_{\varphi}\right) \leq m\left(\pi_{0}\left(D_{\varphi}\right) \cap u^{-1}\left(\pi_{1}\left(D_{\varphi}\right)\right)\right) \leq m\left(\pi_{0}\left(D_{\varphi}\right)\right)=0,
$$

which implies $m\left(\Omega_{\varphi}\right)=0$. Let now $i=1$. From [4, Lemma 1] we have $\nabla u(x)=0$ a.e. in $u^{-1}\left(\pi_{1}\left(D_{\varphi}\right)\right)$ which in other words is

$$
u^{-1}\left(\pi_{1}\left(D_{\varphi}\right)\right) \subset\{x \in \Omega: \nabla u(x)=0\} .
$$

Thanks to [12, Theorem 1.1], we have $y^{\prime}=0$ on $\{x \in \Omega: \nabla u(x)=0\}$, which in particular holds on $u^{-1}\left(\pi_{1}\left(D_{\varphi}\right)\right)$, taking into account (4.3). Since $y^{\prime} \in(\alpha, \beta) \not \supset 0$, this is possible if and only if $m\left(u^{-1}\left(\pi_{1}\left(D_{\varphi}\right)\right)\right)=0$. From (4.2) we then have

$$
m\left(\Omega_{\varphi}\right) \leq m\left(\pi_{0}\left(D_{\varphi}\right) \cap u^{-1}\left(\pi_{1}\left(D_{\varphi}\right)\right)\right) \leq m\left(u^{-1}\left(\pi_{1}\left(D_{\varphi}\right)\right)\right),
$$

which implies $m\left(\Omega_{\varphi}\right)=0$. Hence the thesis follows.

Suppose now that $\tilde{\psi}$ is non constant and choose $t_{1}, t_{2} \in[0,1]$ such that

$$
\tilde{\psi}\left(t_{1}\right)=\min _{t \in[0,1]} \tilde{\psi}(t) \quad \text { as well as } \quad \tilde{\psi}\left(t_{2}\right)=\max _{t \in[0,1]} \tilde{\psi}(t) .
$$

Obviously, $t_{1} \neq t_{2}$ and there is no loss of generality in assuming $t_{1}<t_{2}$. Let $h: \tilde{\psi}([0,1]) \rightarrow[0,1]$ be defined by

$$
h(r)=\min \left(\tilde{\psi}^{-1}(r) \cap\left[t_{1}, t_{2}\right]\right), \quad \forall r \in \tilde{\psi}([0,1]) .
$$

We claim that $h$ is strictly increasing. Indeed, let $r_{1}, r_{2} \in \tilde{\psi}([0,1])$ be such that $r_{1}<r_{2}$. Then, $h\left(r_{1}\right) \neq h\left(r_{2}\right)$ and $t_{1}<h\left(r_{2}\right)$. Taking into account that $\tilde{\psi}\left(h\left(r_{2}\right)\right)=r_{2}>r_{1}, \tilde{\psi}\left(t_{1}\right) \leq r_{1}$, and the continuity of $\tilde{\psi}$, we immediately infer $h\left(r_{1}\right)<h\left(r_{2}\right)$.

Therefore, the family $D_{k}$ of all discontinuity points of the function $k: \mathbb{R} \rightarrow(\alpha, \beta)$ given by

$$
k(r)= \begin{cases}\lambda\left(h\left(\tilde{\psi}\left(t_{1}\right)\right)\right) & \text { if } r \in\left(-\infty, \tilde{\psi}\left(t_{1}\right)\right) \\ \lambda(h(r)) & \text { if } r \in \tilde{\psi}([0,1]) \\ \lambda\left(h\left(\tilde{\psi}\left(t_{2}\right)\right)\right) & \text { if } r \in\left(\tilde{\psi}\left(t_{2}\right),+\infty\right)\end{cases}
$$

is at most countable. Owing to hypotheses (ii) and (iii), this implies that the set

$$
D=D_{\varphi} \cup\left\{\bigcup_{r \in D_{k}}\left[\varphi^{-1}(r) \backslash \operatorname{int}\left(\varphi^{-1}(r)\right)\right]\right\}
$$

has measure zero.

Define now $f: S \rightarrow \mathbb{R}$ by $f(x, z):=k(\varphi(x, z))$. Since $f(S) \subset \lambda([0,1])$ it follows that $f$ is bounded. Moreover, arguing as in [16, Theorem 3.1] gives 
that $f$ is continuous. Set now

$$
F(x, z):=\overline{\mathrm{co}}\left(\bigcap_{\delta>0} \bigcap_{E \in \mathcal{E}} \overline{f\left(B_{\delta}(x, z) \backslash E\right)}\right),
$$

where

$$
\begin{aligned}
\mathcal{E} & =\{E \subset S: m(E)=0\} \\
\text { and } \quad B_{\delta}(x, z) & =\left\{\left(x^{\prime}, z^{\prime}\right) \in S:\left|x-x^{\prime}\right|+\left|z-z^{\prime}\right| \leq \delta\right\} .
\end{aligned}
$$

A standard argument (see, e.g, [16, Theorem 3.1]), ensures that $F$ is upper semicontinuous, with nonempty, convex, and closed values. Furthermore, $F(\cdot, z)$ is measurable for every $z \in \mathbb{R}, F(x, \cdot)$ has a closed graph for almost all $x \in \Omega$, and it holds

$$
F(x, z)=\{f(x, z)\} \quad \text { as soon as }(x, z) \in S \backslash D .
$$

Consider now the problem

$$
-\Delta_{p} u \in F(x, u) \text { in } \Omega, \quad u \in W_{0}^{1, p}(\Omega) .
$$

We want to show existence of solutions to (4.5) by means of Theorem 1.3. To this end, let us verify hypotheses $\left(i_{1}\right)-\left(i_{4}\right)$. If $A_{p}$ is the operator given in (2.3), we choose

$$
U:=A_{p}^{-1}\left(L^{p^{\prime}}(\Omega)\right), \quad \Phi(u):=u \quad \text { and } \quad \Psi(u):=A_{p}(u),
$$

for every $u \in U$. Observe in particular that $A_{p}: U \rightarrow L^{p^{\prime}}(\Omega)$ is bijective.

Let $v_{h} \rightarrow v$ in $L^{p^{\prime}}(\Omega)$. Since $\left\{v_{h}\right\}$ is bounded in $L^{p^{\prime}}(\Omega)$, and $L^{p^{\prime}}(\Omega)$ compactly embeds in $W^{-1, p^{\prime}}(\Omega)$, there exists a subsequence, still denoted by $\left\{v_{h}\right\}$, such that $v_{h} \rightarrow v$ in $W^{-1, p^{\prime}}(\Omega)$. Property $\left(p_{2}\right)$ implies that $A_{p}^{-1}$ is strongly continuous, and therefore $A_{p}^{-1}\left(v_{h}\right) \rightarrow A_{p}^{-1}(v)$ almost everywhere in $\Omega$.

Let now $g: \mathbb{R}_{0}^{+} \rightarrow \mathbb{R}_{0}^{+}$be defined by

$$
g(t):=a(b t)^{1 /(p-1)} \quad \forall t \in \mathbb{R}_{0}^{+},
$$

where the constants $a$ and $b$ come from inequalities (2.1)-(2.2). Note in particular that (2.1) holds true, since by assumption $p>N$. Clearly, $g$ is monotone increasing in $\mathbb{R}_{0}^{+}$. Moreover, fix $u \in U$. Then property $\left(p_{3}\right)$ gives

$\|u\|_{\infty} \leq a\|u\|=a\left\|A_{p}(u)\right\|_{W^{-1, p^{\prime}}(\Omega)}^{1 /(p-1)} \leq a\left(b\left\|A_{p}(u)\right\|_{p^{\prime}}\right)^{1 /(p-1)}=g\left(\left\|A_{p}(u)\right\|_{p^{\prime}}\right)$.

This shows $\left(i_{1}\right)$. Since hypotheses $\left(i_{2}\right)$ and $\left(i_{3}\right)$ are already satisfied, we have only to check $\left(i_{4}\right)$. Define, for every $x \in \Omega$,

$$
\rho(x):=\sup _{|z| \leq g(r)} d(0, F(x, z)) .
$$

Reasoning as in [15, Theorem 3.1], we see that $\|\rho\|_{p^{\prime}} \leq r$ once the same property holds true for the function $x \mapsto j(x):=\sup _{|z| \leq g(r)}|f(x, z)|$.

If $|z| \leq g(r)$, then

$$
\int_{\Omega}|f(x, z)|^{p^{\prime}} \mathrm{d} x \leq m(\Omega)\|f(\cdot, z)\|_{\infty}^{p^{\prime}},
$$


whence

$$
\int_{\Omega}|j(x)|^{p^{\prime}} \mathrm{d} x=\int_{\Omega}\left(\sup _{|z| \leq g(r)}|f(x, z)|\right)^{p^{\prime}} \mathrm{d} x \leq m(\Omega)\|f(\cdot, z)\|_{\infty}^{p^{\prime}} .
$$

Choosing $r \geq m(\Omega)^{1 / p^{\prime}}\|f(\cdot, z)\|_{\infty}$ gives $j \in L^{p^{\prime}}(\Omega)$ and $\|j\|_{p^{\prime}} \leq r$, and hence hypothesis $\left(i_{4}\right)$ is satisfied.

Thanks to Theorem 1.3 there exists $u \in U \subset W_{0}^{1, p}(\Omega)$ such that

$$
-\Delta_{p} u(x) \in F(x, u(x)) \quad \text { a.e. in } \Omega
$$

and $\left|\Delta_{p} u(x)\right| \leq \rho(x)$ for almost every $x \in \Omega$. Define $\Omega_{f}:=\{x \in \Omega$ : $(x, u(x)) \in D\}$. From (4.4) it follows that

$$
\begin{aligned}
\Omega_{f} \subset\left\{\pi_{0}\left(D_{\varphi}\right) \cap u^{-1}\left(\pi_{1}\left(D_{\varphi}\right)\right)\right\} \\
\cup\left\{\bigcup_{r \in D_{k}}\left[\pi_{0}\left(\varphi^{-1}(r) \backslash \operatorname{int}\left(\varphi^{-1}(r)\right)\right) \cap u^{-1}\left(\pi_{1}\left(\varphi^{-1}(r) \backslash \operatorname{int}\left(\varphi^{-1}(r)\right)\right)\right)\right]\right\},
\end{aligned}
$$

which, in particular, implies that

$$
\begin{aligned}
m\left(\Omega_{f}\right) \leq & m\left(\pi_{0}\left(D_{\varphi}\right) \cap u^{-1}\left(\pi_{1}\left(D_{\varphi}\right)\right)\right) \\
& +m\left(\bigcup_{r \in D_{k}}\left[\pi_{0}\left(\varphi^{-1}(r) \backslash \operatorname{int}\left(\varphi^{-1}(r)\right)\right) \cap u^{-1}\left(\pi_{1}\left(\varphi^{-1}(r) \backslash \operatorname{int}\left(\varphi^{-1}(r)\right)\right)\right)\right]\right) \\
\leq & m\left(\pi_{0}\left(D_{\varphi}\right) \cap u^{-1}\left(\pi_{1}\left(D_{\varphi}\right)\right)\right) \\
& +\bigcup_{r \in D_{k}} m\left(\left[\pi_{0}\left(\varphi^{-1}(r) \backslash \operatorname{int}\left(\varphi^{-1}(r)\right)\right) \cap u^{-1}\left(\pi_{1}\left(\varphi^{-1}(r) \backslash \operatorname{int}\left(\varphi^{-1}(r)\right)\right)\right)\right]\right) .
\end{aligned}
$$

Assumption (ii) entails $m\left(\pi_{i}\left(D_{\varphi}\right)\right)=0$ for some $i \in\{0,1\}$. Likewise, due to (iii), for each $r \in D_{k}$, there exists $i_{r} \in\{0,1\}$ such that $m\left(\pi_{i_{r}}\left(\varphi^{-1}(r) \backslash\right.\right.$ $\left.\left.\operatorname{int}\left(\varphi^{-1}(r)\right)\right)\right)=0$. Reasoning like in the case when $\tilde{\psi}$ is constant gives $m\left(\Omega_{f}\right)=0$. This implies $F(x, u(x))=\{f(x, u(x))\}$ and on account of (4.6) it follows that

$$
-\Delta_{p} u(x)=f(x, u(x)) \quad \text { a.e. in } \Omega .
$$

We then have

$$
\psi\left(-\Delta_{p} u(x)\right)=\psi(f(x, u(x)))=\psi(k(\varphi(x, u(x))))=\varphi(x, u(x)),
$$

which completes the proof.

Remark 4.2. Hypothesis (iv) and the assumption $0 \notin(\alpha, \beta)$ are essential to obtain the existence of a solution for equations as in (4.1). Below we consider two situations: apparently they are very similar, but one of them admits a solution while the other one doesn't.

Example 4.3. Let $\varphi: \mathbb{R} \rightarrow \mathbb{R}$ be defined by

$$
\varphi(z)= \begin{cases}0 & \text { if } z \neq 0 \\ 1 & \text { if } z=0\end{cases}
$$


and let $\psi:[1,+\infty) \rightarrow \mathbb{R}$ be such that $\psi(y)=y$. Consider the following equation

$$
-\Delta_{p} u=\varphi(u)
$$

Equation (4.7) doesn't have any solution in $W_{0}^{1, p}(\Omega)$. Suppose on the contrary that $u$ is such a solution. Since $\varphi(u) \geq 0$, then from (4.7) we have $-\Delta_{p} u \geq 0$, and the Strong Maximum Principle implies that $u \equiv 0$ or $u>0$. If $u \equiv 0$, then this would imply that $-\Delta_{p} u \equiv 0$, which is in contrast with (4.7). Suppose now that $u>0$. Then, the definition of $\varphi$ implies $-\Delta_{p} u=0$. This fact, together with the boundary condition $\left.u\right|_{\partial \Omega}=0$, implies $u \equiv 0$ which is again impossible.

Observe also that such $\varphi$ is incompatible with the hypotheses of Theorem 4.1 , because in this case hypothesis (iv) and the condition $0 \notin(\alpha, \beta)$ cannot be verified simultaneously.

Fix now $\lambda \in(0,1)$ and consider the function $\tilde{\varphi}: \mathbb{R} \rightarrow \mathbb{R}$ defined by

$$
\tilde{\varphi}(z)= \begin{cases}1 & \text { if } z \neq 0 \\ \lambda & \text { if } z=0 .\end{cases}
$$

In this case both hypothesis (iv) and $0 \notin[1,+\infty)$ are verified, since

$$
\{1\}=\overline{\tilde{\varphi}(\mathbb{R} \backslash\{0\})} \subset \psi([1,+\infty))=[1,+\infty) .
$$

Therefore, Theorem 4.1 gives the existence of a solution $u \in W_{0}^{1, p}(\Omega)$ to (4.7).

Funding Open Access funding enabled and organized by Projekt DEAL.

Open Access. This article is licensed under a Creative Commons Attribution 4.0 International License, which permits use, sharing, adaptation, distribution and reproduction in any medium or format, as long as you give appropriate credit to the original author(s) and the source, provide a link to the Creative Commons licence, and indicate if changes were made. The images or other third party material in this article are included in the article's Creative Commons licence, unless indicated otherwise in a credit line to the material. If material is not included in the article's Creative Commons licence and your intended use is not permitted by statutory regulation or exceeds the permitted use, you will need to obtain permission directly from the copyright holder. To view a copy of this licence, visit http:// creativecommons.org/licenses/by/4.0/.

Publisher's Note Springer Nature remains neutral with regard to jurisdictional claims in published maps and institutional affiliations.

\section{References}

[1] Ahmad, M., Zada, A., Alzabut, J.: Stability analysis of a nonlinear coupled implicit switched singular fractional differential system with $p$-Laplacian. Adv. Differ. Equ. 2019, Article number: 436 (2019) 
[2] Bartuzel, G., Fryszkowski, A.: On the existence of solutions for inclusion $\Delta u \in F(x, \nabla u)$. In: Marz (ed) Proceedings of the fourth conference on numerical treatment of ordinary differential equations, volume $\mathbf{6 5}$ of Seminarberichte/Humboldt-Univ. zu Berlin, Sekt. Mathematik, Berlin (1984)

[3] Brézis, H.: Functional Analysis, Sobolev Spaces and Partial Differential Equations. Universitext. Springer, New York (2011)

[4] Buttazzo, G., Dal Maso, G., De Giorgi, E.: On the lower semicontinuity of certain integral functionals. Atti Accad. Naz. Lincei Cl. Sci. Fis. Mat. Natur. Rend. Lincei (8) Mat. Appl. 74, 274-282 (1983)

[5] Cabada, A., Heikkilä, S.: Implicit nonlinear discontinuous functional boundary value $\varphi$-Laplacian problems: extremality results. Appl. Math. Comput. 129, 537-549 (2002)

[6] Castaing, C., Valadier, M.: Convex Analysis and Measurable Multifunctions. Springer, Berlin (1977)

[7] Cianchi, A., Maz'ya, V.G.: Global Lipschitz regularity for a class of quasilinear elliptic equations. Comm. Partial Differ. Equ. 36, 100-133 (2010)

[8] Heikkilä, S., Seikkala, S.: On singular, functional, nonsmooth and implicit phiLaplacian initial and boundary value problems. J. Math. Anal. Appl. 308, 513$531(2005)$

[9] Kaczynski, T.: Implicit differential equations which are not solvable for the highest derivative. Lecture Not. Math. 1475, 218-224 (1991)

[10] Klein, E., Thompson, A.C.: Theory of Correspondences. Wiley, New York (1984)

[11] Lê, A.: Eigenvalue problems for the $p$-Laplacian. Nonlinear Anal. 64, 1057-1099 (2006)

[12] Lou, H.: On singular sets of local solutions to $p$-Laplace equations. Chin. Ann. Math. 29B(5), 521-530 (2008)

[13] Marano, S.A.: Implicit elliptic differential equations. Set-Valued Anal. 2, 545$558(1994)$

[14] Marano, S.A.: On a Dirichlet problem with $p$-Laplacian and set-valued nonlinearity. Bull. Aust. Math. Soc. 86, 83-89 (2012)

[15] Marano, S.A.: Elliptic boundary-value problems with discontinuous nonlinearities. Set-Valued Anal. 3, 167-180 (1995)

[16] Marano, S.A.: Implicit elliptic boundary-value problems with discontinuous nonlinearities. Set-Valued Anal. 4, 287-300 (1996)

[17] Marano, S.A., Mosconi, S.J.N.: Lower semi-continuous differential inclusions with $p$-Laplacian. Libertas Math. 33(1), 109-123 (2013)

[18] Peral, I.: Multiplicity of solutions for the $p$-Laplacian, ICTP Lecture Notes of the Second School of Nonlinear Functional Analysis and Applications to Differential Equations, Trieste (1997)

[19] Ricceri, B.: Applications de théoremes de semi-continuité inférieure. C.R. Acad. Sci. Paris, Série I 295, 75-78 (1982)

[20] Ricceri, B.: On multifunctions with convex graph. Atti Accad. Naz. Lincei Rend. Cl. Sci. Fis. Mat. Natur. (8) 77, 64-70 (1984)

[21] Shah, K., Hussain, W., Thounthong, P., Borisut, P., Kumam, P., Arif, M.: On nonlinear implicit fractional differential equations with integral boundary 
condition involving $p$-Laplacian operator without compactness. Thai J. Math. 20, 301-321 (2018)

Greta Marino

Technische Universität Chemnitz

Fakultät für Mathematik

Reichenhainer Strasse 41

09126 Chemnitz

Germany

e-mail: greta.marino@mathematik.tu-chemnitz.de

\section{Andrea Paratore}

Dipartimento di Matematica e Informatica

Università degli Studi di Catania

Viale A. Doria 6

95125 Catania

Italy

e-mail: aparatore@dmi.unict.it

Received: May 2, 2020.

Revised: July 14, 2020.

Accepted: February 5, 2021. 